\title{
El trabajo experimental en la enseñanza de la Física en tiempos de pandemia mediante el uso de la aplicación II Ley de Newton en la UNED de Costa Rica
}

\author{
Experimental work in the teaching of Physics in times of pandemic through \\ the use of Newton's $2^{\text {nd }}$ Law app at UNED in Costa Rica
}

Trabalho experimental no ensino de física em tempos de pandemia
usando a Lei Il de Newton na UNED na Costa Rica

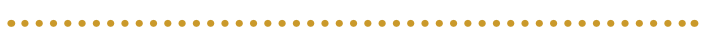

\author{
Eduardo Arias Navarro \\ Universidad Estatal a Distancia \\ San José, Costa Rica \\ earias@uned.ac.cr \\ (D) https://orcid.org/0000-0002-8293-7686 \\ Carlos Arguedas-Matarrita \\ Universidad Estatal a Distancia \\ San José, Costa Rica \\ carguedas@uned.ac.cr \\ (1) https://orcid.org/0000-0003-0939-4627
}

Recibido - Received - Recebido: 24 / 07 / 2020 Corregido - Revised - Revisado: 15 / 09 / 2020 Aceptado - Accepted - Aprovado: 18 / 09 / 2020

DOI: https://doi.org/10.22458/ie.v22iespecial.3204

URL: https://revistas.uned.ac.cr/index.php/innovaciones/article/view/3204

\begin{abstract}
Resumen: La sistematización presenta los resultados del uso del Laboratorio Virtual II Ley de Newton en dos asignaturas de Física de la Universidad Estatal a Distancia de Costa Rica. Este laboratorio permitió realizar aprendizaje experimental sin la necesidad de tener que asistir presencialmente. Se realizó una investigación-acción siguiendo una mediación práctica en la que los participantes deben de realizar una guía de laboratorio por medio de una aplicación. Entre los principales resultados se resalta que los participantes muestran una gran aceptación del Laboratorio Virtual como recurso de aprendizaje del componente experimental, por medio de un dispositivo móvil, y rescatan el valor de este recurso para el logro de aprendizajes en tiempos de pandemia, cuando el aislamiento social ha obligado al cierre instalaciones de las instituciones educativas y se debe mediar el proceso de enseñanza y aprendizaje por medios virtuales.
\end{abstract}

Palabras clave: Física, Laboratorio, Experimento, Mecánica, Pandemia, COVID-19

\begin{abstract}
Here is a systematization that displays the results of using the Virtual Laboratory of Newton's 2nd Law in two of the Physics teaching subjects of Costa Rica's distance learning university (Universidad Nacional a Distancia UNED). This lab enabled experimental learning without the need to go to in-person classes. An actionresearch was carried out, followed by mediation practice in which participants had to execute a lab-guide using an app. Among the primary outcomes is highlighted that the participants were very accepting of the Virtual Lab as a learning resource of the experimental component through a mobile device cultivating the value of this kind of resource to make learning occur in pandemic times while the lockdown has forced the closedown of educational institutions, therefore, mediation in the teaching-learning process is necessary.
\end{abstract}

Key Words: physics, lab, experiment, mechanics, pandemic, COVID-19 
Resumo: A sistematização apresenta os resultados da utilização do Laboratório Virtual II Lei de Newton em duas disciplinas de física da Universidad Estatal a Distancia de Costa Rica. Este laboratório permitiu o aprendizado experimental sem a necessidade de comparecer pessoalmente. Uma pesquisa-ação foi realizada após uma mediação prática na qual os participantes devem fazer um guia de laboratório por meio de uma aplicação. Entre os principais resultados, vale mencionar que os participantes demonstram uma grande aceitação do Laboratório Virtual como recurso de aprendizagem do componente experimental, por meio de um dispositivo móvel, e resgatam o valor deste recurso para a realização da aprendizagem em tempos de pandemia, quando o isolamento social forçou o fechamento de instituições educacionais e o processo de ensino e aprendizagem por meios virtuais deve ser mediado.

Palavras-chave: Física, laboratório, experiência, mecânica, pandemia, COVID-19

\section{INTRODUCCIÓN}

Desde hace varios años la educación ha experimentado grandes cambios influenciados por el avance de las Tecnologías de la Información y la Comunicación (TIC), las cuales han generado una diversidad de recursos educativos en todas las áreas del conocimiento. En el campo específico de la enseñanza de la Física uno de estos avances son los Laboratorios Virtuales (LV). Estos recursos consisten en sistemas programados que permiten simular el comportamiento físico de un fenómeno.

En el contexto actual, la pandemia por el covid-19, dejó claro que un aspecto esencial es la flexibilidad educacional, entendida como las posibilidades de reorganización de la educación en función de los diversos intereses o necesidades (Mill, 2014). En esta línea, las características de los LV se presentan como recursos educativos para fortalecer el trabajo experimental en Física dado el aislamiento social obligatorio, y la imposibilidad de asistir a los edificios educativos.

El trabajo experimental en la enseñanza de la Física es una actividad ineludible que promueve en el estudiante, capacidades diversas y, contribuyen al desarrollo de las competencias requeridas en un profesional de las ciencias y las ingenierías (Arguedas Matarrita, 2017). Por lo tanto "se asume la experimentación como un espacio de construcción de conocimiento, donde se establece una relación dialéctica entre la teoría y la práctica (Espinosa \& Salazar, 2017, p.150). Desde esta óptica se deben buscar espacios y recursos educativos que favorezcan el aprendizaje experimental en tanto se vea disminuida la asistencia a las tutorías presenciales de laboratorio. Máxime en la Universidad Estatal a Distancia (UNED) de Costa Rica, institución líder en educación a distancia en la región. En consecuencia, en esta Institución se han ampliado los espacios experimentales por medio de Laboratorios Remotos (LR) (Arguedas-Matarrita et al, 2019) y los LV de desarrollo propio.

En Latinoamérica se han desarrollado muy pocos LV destinados a la enseñanza de la Física (ArguedasMatarrita, Concari \& Marchisio 2017). Desde hace tiempo, la UNED cuenta con estos recursos educativos para la enseñanza de la Química y la Biología, pero no para la física. Debido a la ausencia de este tipo de laboratorios para la enseñanza de la Física se desarrolló el LV sobre la ll Ley de Newton. Para ello, se conformó equipo multidisciplinario de trabajo en el que participó el Programa de Producción Electrónica Multimedial (PEM) y dos docentes de la cátedra de Física, por parte del PEM se contó con una productora académica, una diseñadora, un artista 3D y dos programadores, el LV se desarrolló en un periodo de 12 meses.

En la etapa de preproducción del LV se conceptualizó que el mismo sería diferente a las simulaciones de acceso libre que ya estaban disponibles, de tal forma que el LV fuese lo más cercano a las prácticas que se realzan en los laboratorios presenciales (Ureña y Arguedas-Matarrita, 2018). Esta planificación permitió que el producto final sea multiplataforma, ya que cuenta con una versión web y una versión aplicación, la versión aplicación cuenta con Realidad Aumentada (RA), lo que permite una mayor inmersión por parte de los usuarios cuando realizan su trabajo experimental. En la figura 1, se muestra la versión aplicación de este LV. 
Figura 1. Aplicación de Segunda Ley de Newton en modo RA.

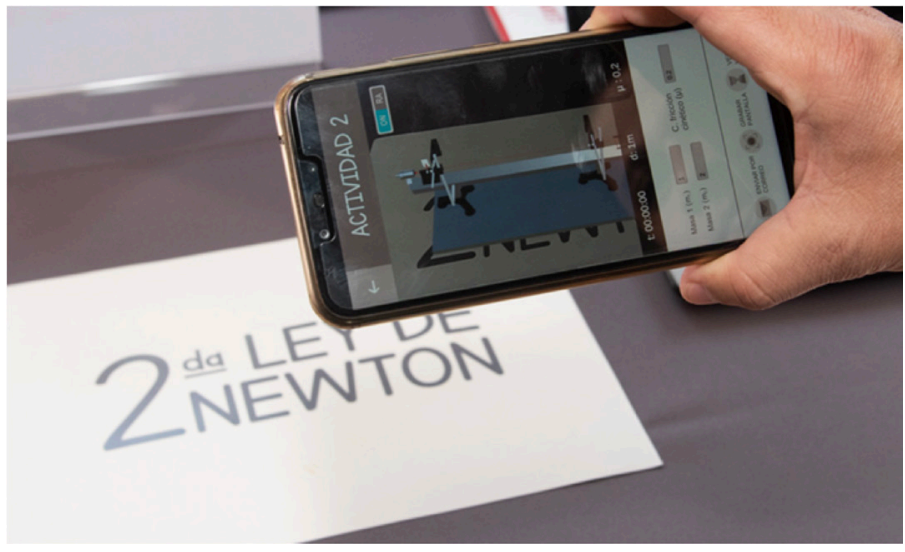

Fuente: https://www.flickr.com/photos/132828733@N06/49609125756/in/album-72157713330135531/

En este LV se puede trabajar tres experimentos diferentes: un plano inclinado donde puede variar la masa, el coeficiente de fricción y el ángulo de inclinación, una segunda actividad en la que los usuarios pueden variar el valor de dos masas unidas por una cuerda, una de ellas colgando por medio de una polea, en este experimento pueden variar las masas y el coeficiente de fricción, y en la tercera actividad se tienen dos masas unidas por una cuerda que pasa por una polea, pero en este caso hay un ángulo de inclinación, en las tres actividades el usuario observa lo que sucede y en la aplicación se registra un tiempo para determinar la aceleración del cuerpo que se mueve o del sistema. Además, se muestran los respectivos Diagramas de Cuerpo Libre y el desarrollo de ecuaciones en cada situación.

El laboratorio inicialmente fue desarrollado para complementar las actividades de la asignatura de Laboratorio de Física General I, pero debido a las medidas de distanciamiento social adoptadas por el gobierno de Costa Rica y aprovechando el potencial del LV que permite realizar prácticas de Movimiento Rectilíneo Uniformemente Acelerado, Leyes de Newton, Trabajo y Energía, se convirtió en un recurso muy valioso para posibilitar la realización de experimentos y así completar las actividades experimentales de dos asignaturas ofrecidas en la UNED.

Por otra parte, el LV tiene un diseño atractivo con una metáfora pedagógica alusiva a la aplicación de las Leyes de Newton a la vida cotidiana. En la figura 2 se muestra el diseño del LV.

Figura 2. Diseño gráfico del LV.

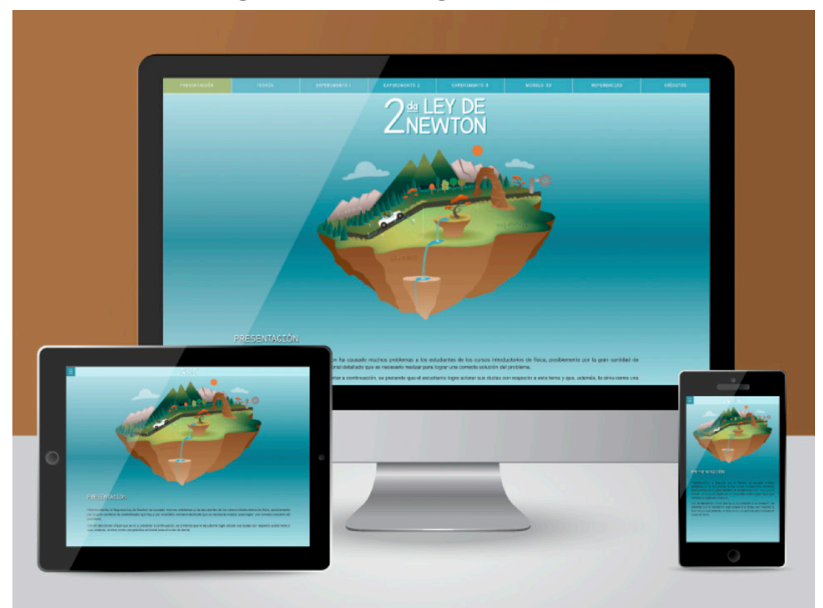

Fuente: Tomado de https://multimedia.uned.ac.cr/index.php?action=detalle\&id=91\#prettyPhoto/0/ 
Algunas de las características que poseen los LV se detallan a continuación:

- Se trabaja con equipos simulados.

- Permiten repetir una experiencia cuantas veces sea necesario.

- Permiten observar de cerca procesos que son difíciles de estudiar en la naturaleza (Monge y Méndez, 2007).

- Permiten detener un experimento en el momento que se desee, por lo que se puede analizar a fondo el comportamiento de determinado fenómeno que dependa del tiempo.

- Facilitan el trabajo experimental cuando no se dispone de espacios ni equipos de laboratorio.

En una investigación reciente Menchaca, Dziabenko y García, 2020 señalan que "las comparaciones entre experimentos simulados y reales demostraron que ambos enfoques podían resultar igual de eficaces en la adquisición del conocimiento" (p. 391), el aprovechamiento de estos recursos se asocia a una buena mediación docente y abordaje pedagógico utilizado.

El objetivo de este trabajo es presentar los resultados de la mediación realizada por medio del LV denominado II Ley de Newton en dos asignaturas universitarias de la UNED para completar el trabajo experimental en momentos donde no se puede asistir a los laboratorios presenciales.

\section{DESARROLLO DE LA EXPERIENCIA: PROPOSICIÓN Y ARGUMENTACIÓN}

Este trabajo se inscribe en el enfoque de investigación cualitativa mediante el tipo de investigaciónacción, donde se realiza una "mediación práctica...colaborativa a fin de mejorar su práctica educativa a través de ciclos de acción y reflexión" (Latorre, 2003, p. 24), es decir, se busca que los estudiantes puedan generar un cambio social y se fomente una conciencia entre ellos "sobre sus circunstancias y la necesidad de mejorar" (Hernández, Fernández y Baptista, 2014, p. 497) hacia este tipo de recursos. Para ello se consideró mediar sobre las siguientes etapas:

- Problemática: Dadas las circunstancias del cierre de los recintos universitarios por "el virus de la COVID-19" la cátedra de Física debió implementar una mejora hacia las asignaturas que contemplaban actividades experimentales y hacer un cambio a la necesidad de cubrir las actividades experimentales propuestas desde un inicio.

- Análisis del plan: Se analizó las posibilidades y recursos que se disponían hasta formular un nuevo proceso o plan de contingencia que buscara resolver el problema presentado.

- Ejecución del plan: Posteriormente, se puso en práctica el plan establecido a fin de recolectar los resultados esperados por la cátedra y facilitar la retroalimentación que se esperaba de estas actividades.

Por tanto, para llevar a cabo esta investigación fue necesaria la recolección de datos, se consideró algunos parámetros estadísticos y otros numéricos sobre la percepción de los estudiantes como herramienta para validar los procesos o etapas propuestas desde la investigación-acción.

Además, cabe mencionar que la investigación se desarrolló con una población de 31 estudiantes matriculados en dos asignaturas de la cátedra de Física de la UNED y para la recolección de los datos se utilizó un instrumento tipo encuesta que fue validado por expertos de la disciplina previamente, por último, los resultaron fueron analizados mediante técnicas estadísticas como la prueba t-student, escala Likert y análisis reflexivo mediante diferentes argumentos brindados por la población estudiantil. A 
continuación, se detalla un poco más del contexto, la muestra y el instrumento de recolección, así como la actividad experimental propuesta.

\section{CONTEXTO}

En este estudio se consideraron dos asignaturas de la cátedra de Física de la UNED de Costa Rica, en las que los estudiantes participantes se encontraban distribuidos en diferentes centros universitarios a lo largo del país (observe la tabla 1), durante el primer cuatrimestre del año 2020. Las asignaturas inicialmente estaban planificadas para realizar cuatro tutorías presenciales de laboratorios, pero debido al aislamiento social y cierre de los recintos con laboratorios, se utilizó la Aplicación II Ley de Newton para lograr cumplir con las actividades experimentales propuestos por la cátedra, para ello se realizaron guías de laboratorio en las que se desarrollaron los objetivos de aprendizaje que se buscaban alcanzar en las sesiones de laboratorio utilizando dicha aplicación.

La primera asignatura en la se utilizó el LV fue Física General para Agroindustrial; esta asignatura se encuentra situada en el primer año de carrera de Ingeniería Agroindustrial en el bloque B y tiene asignado un valor de 3 créditos, contempla en su evaluación 2 parciales de $25 \%$ cada uno, 2 tareas de $15 \%$ cada una, una actividad en línea de $10 \%$ y una práctica virtual de 10\%. Para esta última evaluación se consideró el uso de la aplicación de la II ley de Newton empleando la práctica denominada la II Ley de Newton.

La otra asignatura fue el Laboratorio de Física I para la Enseñanza de las Ciencias, dirigido a estudiantes del profesorado de la carrera de Enseñanza de las Ciencias. Esta asignatura es de 1 crédito y se ubica en el bloque $C$ del segundo año de la carrera, su evaluación consta de la realización de cuatro pre-reportes de $2 \%$ cada uno, cuatro pruebas cortas de $4 \%$ cada una, el trabajo de laboratorio de $12 \%$, dos actividades en línea de $4 \%$ cada una y dos prácticas virtuales o remotas de $8 \%$ cada una. Para esta asignatura, el uso de la Aplicación II Ley de Newton, se realizó durante la práctica 3, denominada II Ley de Newton y la práctica 4 denominada el teorema trabajo-energía.

TABLA 1

Lista de centros universitarios con número de estudiantes que respondieron la encuesta en las asignaturas de Laboratorio de Física I y Física para Agroindustrial

\begin{tabular}{lccccc}
\multicolumn{5}{c}{ Centros universitarios y número de estudiantes } \\
San José & 7 & Pérez Zeledón & 1 & Atenas & 2 \\
Puriscal & 1 & Cartago & 4 & Palmares & 2 \\
Acosta & 2 & Turrialba & 2 & Puntarenas & 1 \\
San Marcos & 3 & San Isidro & 1 & Liberia & 1 \\
Desamparados & 2 & Alajuela & 1 & Guápiles & 1 \\
\hline
\end{tabular}

La asignatura de Física General para Agroindustrial registró una matrícula de 65 estudiantes y la asignatura de Laboratorio de Física General I se contabilizó una matrícula de 26 estudiantes. Para el desarrollo de las asignaturas, los estudiantes utilizaron la plataforma virtual Moodle mediante el enlace Aprende U donde se encuentran las aulas virtuales de las asignaturas que imparte la UNED. 


\section{MUESTRA E INSTRUMENTO DE RECOLECCIÓN DE DATOS}

El instrumento para la recolección de información consistió en un cuestionario autoadministrado, constituido por 20 ítemes de los cuales quince fueron de selección única, dos de selección múltiple y tres preguntas abiertas. Los ítemes se elaboraron para recolectar información sobre: aspectos generales, de conectividad, uso del LV, usabilidad, percepción del aprendizaje y satisfacción con el objetivo de recopilar la información relevante al uso de esta aplicación en medio de la pandemia ocasionada por "el virus responsable de la COVID-19". Cabe destacar, que las respuestas recopiladas representan cerca del 32\% y $38 \%$ de las personas matriculadas al inicio de cada asignatura.

El cuestionario fue validado por especialistas en Física y Educación, los cuales tienen conocimiento en el uso de materiales didácticos y en la plataforma MOODLE, una vez que se realizó las prácticas en las que utilizaron el LV se les envió el instrumento por medio de la plataforma y se obtuvo la respuesta del $32 \%$ de la asignatura para Agroindustrial y $38 \%$ de la asignatura Laboratorio Física Genera I para la Enseñanza de las Ciencias, de las personas matriculadas al inicio de cada asignatura. Lo que representa una muestra constituida por los estudiantes que eligieron responder el cuestionario autoadministrado $(n=31)$.

\section{ACTIVIDAD EXPERIMENTAL PROPUESTA}

Para la actividad de la II Ley de Newton se pretendió que el estudiante determinara la relación entre la aceleración y la masa, pendiente y coeficiente de fricción para un cuerpo que se desplaza por un plano inclinado. Para este caso el estudiante seleccionó la actividad 1, luego en la primera parte los estudiantes variaron la masa del objeto para un ángulo y coeficiente de fricción cinético cero a fin de obtener la aceleración experimental, posteriormente variaron el ángulo con un valor de masa constante y coeficiente de fricción cero y nuevamente se obtuvo la aceleración experimental. Luego, se realizó un último caso donde se varió el coeficiente de fricción cinético a un valor constante de masa y ángulo para conseguir su valor de aceleración experimental. Una vez recopilados los datos de aceleración estos fueron comparados con sus respectivos valores teóricos para comparar los márgenes de error y responder a una serie de preguntas basadas en el principio físico de este movimiento. Cabe destacar que esta práctica fue utilizada en ambas asignaturas.

Por su parte, en la asignatura Laboratorio de Física I para Enseñanza de las Ciencias también se utilizó la aplicación, pero esta vez se realizó la práctica de teorema trabajo-energía a fin de comprobar experimentalmente el teorema al variar el ángulo en un plano inclinado, el coeficiente de fricción cinético de un carro dinámico y la masa colgante del sistema, para este caso se usó la actividad 3 de la aplicación. En esta ocasión se tenía el sistema de dos bloques o masas, para la primera parte se varió el ángulo manteniendo constantes las masas, coeficiente de fricción y la distancia a fin de que se recopilara datos de aceleración, la segunda y tercera parte se varió el coeficiente de fricción y la masa colgante (segundo objeto) respectivamente. En ambos casos se recopiló los datos de aceleración a fin de luego calcular la velocidad final para un desplazamiento marcado por la aplicación. Con esto se logró calcular la energía cinética del sistema y se comparó con el trabajo neto ejercido por el primer objeto. Por último, se compararon los resultados experimentales tanto de la energía cinética como el trabajo neto y se obtuvo un porcentaje de diferencia a fin de justificar el teorema del trabajo-energía, el cual se abordó mediante varias interrogantes que los estudiantes debían contestar en forma de discusión en su respectivo reporte. 


\section{RESULTADOS}

Con el estudio que se llevó a cabo empleando la aplicación de la II Ley de Newton, se establece una clara evidencia de la importancia de los LV en modelos de educación a distancia y cómo este tipo de estrategias novedosas pudieron ser adaptadas de forma exitosa ante la emergencia vivida por el virus responsable de la COVID-19 y cómo esta tuvo una adecuada aceptación por parte de la población estudiantil que participante.

Para los resultados obtenidos en cuanto a aspectos generales, se debe mencionar que para la asignatura de Física General para Agroindustrial los estudiantes tenía un promedio de edad de 25 años, mientras que para la asignatura de Laboratorio de Física General I una edad promedio de 26 años. En cuanto a la conectividad, se destaca cómo al inicio todos los estudiantes de ambas asignaturas tenían clases de forma presencial o tutorías, sin embargo, producto de la declaratoria de la pandemia provocada por el virus de la COVID-19 en Costa Rica, ambas asignaturas fueron reestructurados para culminar sus actividades mediante clases virtuales. Esto hizo que respecto a la conectividad los estudiantes tuvieran nuevos retos que asumir, por ello de los encuestados se obtuvo que un $45 \%$ de los estudiantes señalaron tener mala o poca conexión a Internet, una persona (3\%) sin dispositivos electrónicos como computadora, Tablet o teléfono y por último se registró un 54\% de personas que argumentaron no tener recursos como impresora para poder imprimir el marcador para ser utilizado como realidad aumentada.

En relación con los aspectos de usabilidad y percepción del aprendizaje es importante destacar que, para el instrumento utilizado, se realizó una escala Likert que tuvo la siguiente clasificación por categoría: 1 Totalmente en desacuerdo 2 En desacuerdo 3 Ni en desacuerdo ni de acuerdo 4 De acuerdo 5 Totalmente de acuerdo. Por tanto, en la tabla 3 se presentan los resultados obtenidos.

Además, cabe mencionar que para consolidar la medida general de la escala Likert se aplicó una prueba t-student de varianzas desiguales a los datos recopilados por cada grupo, en dicha prueba se contempló un valor de significancia de $a=0,05$. La tabla 2 resume los datos de la prueba t-student, donde se define $t(15)=2.048 ; p=0,53$, donde $p$ representa el valor de probabilidad con 0.525 . Este valor es mayor a la significancia, lo que permite concluir que las dos medias obtenidas para cada grupo no tienen diferencia significativa, en la tabla 3 se consigna la medida general ambos grupos.

TABLA 2

Prueba t-student para muestras de varianzas desiguales de las medidas de escala Likert

\begin{tabular}{lcc} 
& Grupo de Agroindustrial & Laboratorio de Física I \\
Media & 3,72 & 3,85 \\
Varianza & 0,189 & 0,357 \\
Observaciones & 15 & 15 \\
Varianza agrupada & 0,273 & \\
Diferencia hipotética de las medias & 0 & \\
Grados de libertad & 28 & \\
Estadístico t & $-0,644$ \\
P(T<=t) una cola & 0,262 \\
Valor crítico de t (una cola) & 1,701 & \\
$\mathrm{P}(\mathrm{T}<=\mathrm{t})$ dos colas & 0,525 & \\
Valor crítico de $t$ (dos colas) & 2,048 \\
\hline
\end{tabular}

Fuente: elaboración propia, 2020. 
Respecto a la medida obtenida en la tabla 3, es notorio destacar cómo para los estudiantes el usar la aplicación de la II Ley de Newton representó un medio destacable para el análisis de fenómenos físicos, ya que se comprendió lo que estaba realizando y a su vez el experimento realizado desde casa facilitó ese aprendizaje. Rosado y Herreros, $(2005$, p. 2) calificaron a los LV como una "herramienta de autoaprendizaje y con esto se permite obtener una visión más intuitiva de aquellos fenómenos que en su realización manual". Además, destacaron como favorable que el uso de LV da lugar a cambios fundamentales en el proceso habitual de enseñanza.

TABLA 3

Resultados de escala Likert

\begin{tabular}{lc}
\multicolumn{1}{c}{ Criterios evaluados } & Medida \\
\hline La instalación de la aplicación fue sencilla. & 4,6 \\
El tiempo de adaptación para usar la aplicación dificultó realizar el experimento. & 2,5 \\
Me sentí identificado con la actividad realizada en la aplicación. & 3,6 \\
El uso del marcador de realidad aumentada mejora la experiencia experimental. & 3,4 \\
La aplicación fue muy simple usar un Laboratorio Virtual (LV). & 3,9 \\
Las guías de laboratorio son claras. & 3,4 \\
Los datos obtenidos en la aplicación me permitieron alcanzar el objetivo planteado. & 3,9 \\
Al trabajar en la aplicación comprendí lo que estaba haciendo. & 4,1 \\
Al trabajar en la aplicación pude comprobar el principio físico que se buscaba. & 3,9 \\
Las habilidades adquiridas fueron valiosas en su aprendizaje. & 3,9 \\
El uso de la aplicación me permite realizar actividades similares a las del laboratorio presencial. \\
Me gustaría usar este tipo de recurso educativo en otras asignaturas. \\
El uso de la aplicación es importante en la educación a distancia. \\
La posibilidad de realizar un experimento en la casa por medio de la aplicación facilitó su aprendizaje. \\
En general estoy satisfecho con el uso de la aplicación.
\end{tabular}

Fuente: elaboración propia, 2020.

Otro aspecto relevante se da con el perfil que presenta este tipo de recursos para la modalidad que ofrece la UNED, vemos cómo uno de los puntos favorables en la medida fue que los estudiantes califican la aplicación como un insumo importante para la educación a Distancia y como una herramienta innovadora en las prácticas docentes (Liete, Maidana, Fonseca y Vanin, 2015).

Por otra parte, es importante no omitir que parte de los retos que se asumen con este tipo de recursos como son los LV y propiamente la aplicación de la II Ley de Newton es que los docentes puedan acompañar más en el uso de los LV (González, Escobar, Beltrán, García \& Hoz, 2019). En este caso particular, la medida más baja con el 2.5 resaltó cómo los estudiantes, aun cuando el recurso tiene muchas ventajas requieren de una guía para comprender en su totalidad el uso de estos. En este sentido Arias \& Arguedas (2018), destacan cómo es de vital importancia la realización de talleres y capacitaciones introductorias a los docentes para optimizar el uso este tipo de recursos.

En cuanto a la satisfacción de esta aplicación es importante destacar los siguientes comentarios que se dieron por parte del estudiantado, algunas de esas afirmaciones se reflejan en la tabla 4, donde el principal aporte es la importancia de incorporar este tipo de recursos tanto en el ámbito de la modalidad de la educación a distancia, como por la tendencia de las nuevas tecnologías para los procesos de enseñanza y aprendizaje del siglo XXI (Lestari y Supahar, 2020). 
Además, lo acontecido con la pandemia provocada por el "el virus responsable de la COVID-19" evidenció que este tipo de recursos facilitaron la transición de las tutorías presenciales al trabajo de laboratorio virtual, siendo algo de relevancia para continuar con un adecuado proceso de enseñanza, tal y como exponen los mismos estudiantes en algunas afirmaciones.

TABLA 4

Afirmaciones positivas de los estudiantes respecto a la aplicación

\begin{abstract}
Afirmaciones
Son realmente muy sencillos de utilizar, además de entretenidos, se debería implementar siempre

Es una muy buena opción, así no se pierde la materia ni el tiempo. Usar la tecnología en momentos de crisis como lo estamos haciendo en estos días ayuda a mejorar el aprendizaje.

Cuando las instrucciones para el uso de la aplicación, así como el objetivo del por qué se está implementando son claras, este tipo de aplicaciones son de gran ayuda para el estudiante a distancia ya que facilita un poco el aprendizaje y el desarrollo de actividades que antes solo se podían realizar en un laboratorio, y ahora con este tipo de facilidades se pueden desarrollar desde algún lugar con disposición a Internet lo que es de mucha utilidad.
\end{abstract}

Me parecen una buena alternativa para remplazar las actividades experimentales presenciales que fueron suspendidas. Pienso que sí es posible abarcar los diferentes temas que ameritan trabajo experimental por medio de este tipo de herramientas.

Es de suma importancia ya que permiten realizar actividades similares a las de un laboratorio presencial, que nos permiten comprender lo que se está haciendo y estudiando.

La valoro como buena, ya que la aplicación ayudó a brindar la experiencia de un laboratorio de Física, el cual ayuda a que el aprendizaje sea más optimo, y se pueda entender mejor la materia.

Es una herramienta muy practica que ayuda a comprender mejor los conceptos con los que se está trabajando durante el curso. Se debería usar en más cursos, ojalá con instrucciones más claras de cómo utilizar la app.

Personalmente me parece una herramienta tanto útil como indispensable, ya que le permite al estudiante tener un apoyo al estudiar. La posibilidad de realizar experimentos desde casa es algo importante en la educación a distancia. Y en situaciones de pandemia, como la que se atraviesa actualmente, es una buena opción para la sustitución de los laboratorios presenciales.

Es una herramienta práctica, que permite a uno como estudiante a estar más familiarizado con la materia, entender ciertos puntos, que sólo con la teoría no son tan claros, sí estoy de acuerdo que se siga implementando este tipo de actividades virtuales y ojalá que la universidad pueda en algún momento contar con laboratorios de Física presenciales, serían de gran nutrición académica para nosotros como estudiantes, pero por el momento estos laboratorios virtuales han funcionado muy bien en el aprendizaje

Me parece excelente, ya que podemos continuar con nuestro aprendizaje desde la casa

Es un recurso que le permite al estudiante poner en práctica los conocimientos adquiridos, seguir enriqueciendo el aprendizaje, además es una herramienta fácil de usar.

Este fue un actuar muy rápido que este ayudó a que no se fragmentara el proceso de enseñanza-aprendizaje

Fuente: elaboración propia, 2020.

De igual forma, el uso de esta aplicación representó limitantes en algunos aspectos por parte del estudiante, la tabla 5 resume algunos de los comentarios con aspectos por mejorar que son de vital importancia para poder valorar nuevos aportes y desarrollar nuevos productos que atiendan este tipo de necesidades.

Entre estas limitaciones están que el docente intervenga más en el proceso educativo de este tipo de laboratorios, se brinde un material que sea elaborado con base en el contexto cultural donde se desenvuelve el estudiantado y se tenga consideración de las oportunidades que tiene el estudiante con base en los recursos que este necesita. 
TABLA 5

Afirmaciones de aspectos por mejorar por parte de los estudiantes respecto al uso de la aplicación

\section{Afirmaciones}

Sí creo que deben tener mayor acompañamiento a los estudiantes en estos procesos, que los profesores tengan mayor acercamiento a los estudiantes, ya que a muchos nos cuesta manejar algunas aplicaciones.

Es una alternativa para poder continuar con los trabajos y comprender un poco mejor la aplicación de la materia, considero que se debería realizar un video con la descripción del uso adecuado de la herramienta y sus diferentes funciones.

El laboratorio virtual me parece una buena estrategia debido a la situación que estamos enfrentando, sin embargo, jamás se comparan con laboratorios presenciales.

Por la emergencia mundial sí es un buen recurso. Sin embargo, siento que se le podría sacar más provecho a la app como incluir nuevas características con un poco más de guía de parte de los profesores y asi aprovecharla lo más parecido a un laboratorio presencial.

Es útil ya que me permite visualizar el experimento. En la guía nunca se nos explicó cómo se usa la realidad aumentada y por lo mismo no fue de relevancia en el trabajo ya que no se utilizó.

Me parece muy buena su implementación, lastimosamente no todos tenemos la misma disponibilidad para acceder al Internet más que todo los de zonas rurales que como en mi caso solo se depende del Internet de celular. Por lo demás está excelente y me gustó.

Importante, porque al no poder asistir a las sesiones presenciales este tipo de aplicaciones son muy parecidas a uno real, además se evita salir de la casa, entonces si sirve de mucho, el único problema es que hay personas que no cuentan con Internet en casa, pero por lo demás es muy buena.

Es una gran ayuda para no exponer a la comunidad universitaria. Se apreciaría tomar en cuenta este tipo de aplicaciones para los cursos de Física II y III

La intención de utilizar la App es muy buena, al igual que la facilidad de su uso. Me agradó bastante. Sin embargo, las indicaciones que venían en la app, las de las prácticas e incluso la de los cuadros no coincidían mucho o no tenían mucha claridad, lo que dificultó comprender cuáles eran los datos que tenía que usar para completar la actividad.

Fuente: elaboración propia, 2020.

También se destacó que se debe potenciar el acompañamiento docente, el cual debe ser más oportuno, además se requiere diseñar videos explicativos para aprovechar más el LV, en este sentido se debe tener en cuenta que en un principio este recurso no fue pensado para utilizarse en estas asignaturas, y su uso se debió a la pandemia, por lo que estos aspectos de mejora serán tomados en cuenta para las próximas ofertas en las que se utilizará el LV.

\section{SÍNTESIS Y REFLEXIONES FINALES}

El uso de aplicaciones como es el caso de la II Ley de Newton es de vital importancia en el ambiente educativo, esto le permite al docente disponer de nuevas herramientas e incide directamente en que los estudiantes desarrollen no solo aspectos teóricos de un postulado físico, sino que puedan de igual forma desarrollar habilidades en la parte experimental (Lestari y Supahar, 2020). Además, dado el escenario de la pandemia, es de vital importancia destacar cómo este tipo de metodologías fueron bien recibidas por parte de los estudiantes de la UNED para poder continuar con el proceso de enseñanza y aprendizaje que realizaban en el momento que se dio la declaratoria generada por "el virus responsable de la COVID-19", siendo un recurso que no solo es funcional para la educación a distancia, sino también muy bien visto y de fácil adaptación para sobrellevar circunstancias como la vivida mundialmente por la pandemia en otras instituciones académicas.

Este tipo de recurso educativo resultó muy útil para lograr completar las actividades experimentales en las dos asignaturas en los que se usó, la aplicación tiene una gran adaptabilidad a diversas asignaturas y diversos contextos, lo que le confiere un alto potencial educativo no solo para las asignaturas de la UNED, sino de otras instituciones en el nivel secundario y universitario, en este sentido es un recurso 
pedagógicamente agnóstico (Gillet \& Salzmann, 2011) ya que es útil para diferentes niveles y contextos, solo se requiere de una adecuada mediación docente.

Por otra parte, frente a las limitaciones encontradas, no se puede evadir la importancia que tiene la mediación docente en la implementación de estos recursos educativos, se considera relevante disponer de un material acorde a las necesidades de la población estudiantil y la constante mediación por parte del docente durante el proceso del quehacer educativo, no se puede asumir que, a pesar de la facilidad que tienen este tipo de aplicaciones, el estudiante deba explorar por sus propios medios un correcto uso y aplicación para desarrollar su propio laboratorio.

\section{AGRADECIMIENTOS}

Agradecemos primeramente a la Escuela de Ciencias Exactas y Naturales (ECEN) de la UNED por su apoyo a este tipo de trabajos y a la encargada de la cátedra de Física Diana Herrero Villareal por su colaboración en permitir el uso esta aplicación en las asignaturas.

\section{REFERENCIAS}

Arguedas-Matarrita, C.; Orduña, P.; Concari, S.; Elizondo, F.U.; Rodríguez Gil, L.; Hernández, U.; Carlos, L.M.; Conejo-Villalobos, M.; da Silva, J.B.; García Zubia, J. \& da Mota Alves, J.B. (2019). Remote experimentation in the teaching of physics in Costa Rica: First steps. En Proceedings of the 2019, $5^{\text {th }}$ Experiment@ International Conference (exp.at'19), Madeira, Portugal, 12-14 June 2019.

Arguedas Matarrita, C. (2017). Diseño y desarrollo de un Laboratorio Remoto para la enseñanza de la física en la UNED de Costa Rica. (Tesis doctoral). Universidad Nacional de Litoral, Santa Fe, Argentina. Recuperado de https://bibliotecavirtual.unl.edu.ar:8443/bitstream/handle/11185/1018/Tesis. pdf?sequence $=1 \&$ isAllowed $=y$

Arguedas-Matarrita C. Concari, S. B. \& Marchisio, S.T. (2017). Una revisión sobre desarrollo y uso de Laboratorios Virtuales y Laboratorios Remotos en la Enseñanza de la Física en Latinoamérica. En: I Simposio Ibero-Americano de Tecnologías Educativas, Araranguá, Santa Catarina, Brasil, 8 al 10 de mayo, 2017.

Arias, E., y Arguedas-Matarrita, C. (2018). Fortaleciendo la enseñanza de la física en un Colegio Científico Costarricense mediante el uso del Laboratorio Remoto VISIR. Virtualidad, Educación y Ciencia, 9(16), 131-141. Recuperado de https://revistas.psi.unc.edu.ar/index.php/vesc/article/ view/20478/20136

Espinosa, E. y Salazar, T. (2017). Análisis a la experimentación en torno a la herencia genética de Mendel en libros de texto universitarios. En Prácticas experimentales en textos universitarios. Implicaciones en la enseñanza de las Ciencias Naturales. Programa Editorial, Universidad del Valle, Colombia.

González, J. D., Escobar, J. H., Beltrán, J. R., García-Gómez, L., y De La Hoz, J. (2019). Virtual laboratories of electromagnetism for education in engineering: A perception. In Journal of Physics: Conference Series (Vol. 1391, No. 1, p. 012157). IOP Publishing. https://doi.org/10.1088/1742-6596/1391/1/012157

Hernández, R. Fernández, C. y Baptista, M. P. (2014). Metodología de la Investigación, Mc Graw Hill, México

Latorre, A. (2003). La Investigación-Acción. Conocer y Cambiarla. Práctica Educativa. Gro. Barcelona.

Liete, M; Maidana, N; Fonseca, M y Vanin, V. (2015). Innovaciones del Laboratorio Virtual: el experimento de colisiones bidimensionales. Revista de Enseñanza de la Física. Volumen 27 (2). 543-549. Recuperado de https://revistas.unc.edu.ar/index.php/revistaEF/article/view/12703/12941 
Lestar, D; y Supahar. (2020). Student and Teachers' necessity toward virtual laboratory as an instructional media of $21^{\text {st }}$ century science learnig. Journal of Physics: Conference Series 1440 012091. https:// doi.org/10.1088/1742-6596/1440/1/012091

Menchaca, I., Dziabenko, O., y Zubía, J. (2020). Experiencia española en el proyecto GoLab. Educar, 56(2), 387-405. Recuperado de https://educar.uab.cat/article/viewFile/ v56-n2-menchaca-dziabenko-garcia/1067-pdf-es

Mill, D. (2014). Flexibilidade educacional na cibercultura: analisando espaços, tempos e currículo em produções científicas da área educacional. RIED. Revista Iberoamericana de Educación a Distancia, 17(2), 97-126. Recuperado de https://www.redalyc.org/pdf/3314/331431248006.pdf

Monge, J., y Méndez, V. (2007). Ventajas y desventajas de usar laboratorios virtuales en educación a distancia: la opinión del estudiantado en un proyecto de seis años de duración, Revista Educación, vol. 31, núm. 1, pp. 91-108. Recuperado de https://www.redalyc.org/pdf/440/44031106.pdf

Rosado, L., y Herreros, J. (2005). Nuevas aportaciones didácticas de los laboratorios virtuales y remotos en la enseñanza de la Física. Research Developments in Learning Technologies, 1(1), 1-5. Recuperado de https://www.uv.es/eees/archivo/286.pdf

Ureña, F., y Arguedas-Matarrita, C. (2018). Diseño de laboratorios virtuales para la enseñanza de la física. Revista Internacional de Aprendizaje, 5(1), 55-64. https://doi.org/10.18848/2575-5544/CGP/ v05i01/55-64 